\title{
A Short Proof of the Rook Reciprocity Theorem
}

\author{
Timothy Chow \\ Dept. of Mathematics, Univ. of Michigan, Ann Arbor, MI 48109, U.S.A. \\ email: tchow@umich.edu
}

Submitted: February 12, 1996; Accepted: March 4, 1996.

\begin{abstract}
Rook numbers of complementary boards are related by a reciprocity law. A complicated formula for this law has been known for about fifty years, but recently Gessel and the present author independently obtained a much more elegant formula, as a corollary of more general reciprocity theorems. Here, following a suggestion of Goldman, we provide a direct combinatorial proof of this new formula.
\end{abstract}

MR primary subject number: 05A19

MR secondary subject numbers: 05A05, 05A15

A board $B$ is a subset of $[d] \times[d]$ (where $[d]$ is defined to be $\{1,2, \ldots, d\}$ ) and the rook numbers $r_{k}^{B}$ of a board are the number of subsets of $B$ of size $k$ such that no two elements have the same first coordinate or the same second coordinate (i.e., the number of ways of "placing $k$ non-taking rooks on $B$ "). It has long been known [5] that the rook numbers of a board $B$ determine the rook numbers of the complementary board $\bar{B}$ (defined to be $([d] \times[d]) \backslash B)$ according to the polynomial identity

$$
\sum_{k=0}^{d} r_{k}^{B}(d-k) ! x^{k}=\sum_{k=0}^{d}(-1)^{k} r_{k}^{\bar{B}}(d-k) ! x^{k}(x+1)^{d-k}
$$

Recently, a simpler formulation of this identity was found independently by Gessel [2] and Chow [1]. To state it, we follow [4] in defining

$$
R(B ; x) \stackrel{\text { def }}{=} \sum_{k=0}^{d} r_{k}^{B} x \underline{d-k},
$$

where $x \stackrel{n}{\text { def }}=x(x-1)(x-2) \cdots(x-n+1)$. Then we have the following reciprocity theorem.

Theorem. For any board $B \subset[d] \times[d]$,

$$
R(\bar{B} ; x)=(-1)^{d} R(B ;-x-1) .
$$

The existing proofs derive this as a corollary of other reciprocity theorems, but Goldman [3] has suggested that a direct combinatorial proof ought to be possible. Indeed, it 
is, and the purpose of this note is to provide such a proof. The knowledgeable reader will recognize that the main idea is borrowed from [4].

Proof. Observe that

$$
\begin{aligned}
(-1)^{d} R(B ;-x-1) & =(-1)^{d} \sum_{k=0}^{d} r_{k}^{B}(-x-1) \frac{d-k}{} \\
& =\sum_{k=0}^{d}(-1)^{k} r_{k}^{B}(x+d-k) \frac{d-k}{} .
\end{aligned}
$$

First assume $x$ is a positive integer. Add $x$ extra rows to $[d] \times[d]$. Then $r_{k}^{B}(x+d-k) \frac{d-k}{2}$ is the number of ways of first placing $k$ rooks on $B$ and then placing $d-k$ more rooks anywhere (i.e., on $B, \bar{B}$ or on the extra rows) such that no two rooks can take each other in the final configuration. By inclusion-exclusion, we see that the resulting configurations in which the set $S$ of rooks on $B$ is nonempty cancel out of the above sum, because they are counted once for each subset of $S$, with alternating signs. Thus what survives is the set of placements of $d$ non-taking rooks on the extended board such that no rook lies on $B$. But it is clear that this is precisely what $R(\bar{B} ; x)$ enumerates. Therefore the theorem holds for all positive integers $x$ and since it is a polynomial equation it holds for all $x$.

\section{Acknowledgments}

This work was supported in part by a National Science Foundation Graduate Fellowship and a National Science Foundation Postdoctoral Fellowship.

\section{References}

[1] T. Chow, The path-cycle symmetric function of a digraph, Advances in Math., in press.

[2] I. M. Gessel, personal communication.

[3] J. R. Goldman, personal communication.

[4] J. R. Goldman, J. T. Joichi, and D. E. White, Rook theory I. Rook equivalence of Ferrers boards, Proc. Amer. Math. Soc. 52 (1975), 485-492.

[5] J. Riordan, "An Introduction to Combinatorial Analysis," Wiley, New York, 1958. 